\title{
Effects of sulfate starvation on agar polysaccharides of Gracilaria species (Gracilariaceae, Rhodophyta) from Morib, Malaysia
}

\begin{abstract}
The effects of sulfate starvation on the agar characteristics of Gracilaria species was investigated by culturing two red algae from Morib, Malaysia, Gracilaria changii and Gracilaria salicornia in sulfate-free artificial seawater for 5 days. The seaweed samples were collected in October 2012 and March 2013, periods which have significant variation in the amount of rainfall. The agar yields were shown to be independent of sulfate availability, with only $0.60-1.20 \%$ increment in treated G. changii and $0.31-1.40 \%$ increment in treated G. salicornia while their gel strengths did not increase significantly (approximately 5-7 \%) after sulfate starvation for both species. The gelling and melting temperatures did not vary between control and treated samples from both species, except for the treated G. changii collected in March 2013. The gel syneresis index of G. salicornia collected in March 2013 increased significantly after sulfate deprivation. Sulfate starvation introduced some variations in the content of 3,6-anhydrogalactose and total sulfate esters, but the changes did not have a pronounced effect on the physical properties of agar.
\end{abstract}

Keyword: Agar yield; Gel strength; Gracilaria changii; Gracilaria salicornia; Sulfate starvation 